\title{
Cisj
}

\section{REMOVAL OF IMPULSE BURSTS IN SATELLITE IMAGES}

\author{
Oleg Tsymbal ${ }^{1)}$, Volodymyr Lukin ${ }^{1)}$, Pertti Koivisto ${ }^{2)}$, Volodymyr Melnik ${ }^{2)}$
}

1) Dept 504, National Aerospace University (KhAI) 17 Chkalova Street, 61070, Kharkov, Ukraine, Tel/fax +38 0572 441186, E-mail: lukin@xai.kharkov.ua,

2) Institute of Signal Processing, Tampere University of Technology, P.O.Box-553, FIN-33101, Tampere, Finland, E-mail: peko@cs.tut.fi, melnik@cs.tut.fi

\begin{abstract}
Characteristics of impulse bursts in satellite images are analyzed and methods for burst removal are considered. Artificial compact burst model is proposed and test images are created. An advanced multipass algorithm for the detection and removal of compact bursts in the presence of both additive and multiplicative noise is proposed. The efficiency of the algorithm is evaluated quantitatively using the artificial test images and visually using the artificial test images and real radar and optical satellite images. It is shown through experiments that the proposed method removes impulse bursts efficiently while preserving information.
\end{abstract}

Keywords: compact impulse bursts, impulse noise removal, robust filtering, satellite image processing

\section{INTRODUCTION}

Impulsive noise is one of the factors that degrade the quality and interpretation accuracy (especially automatic interpretation) of remote sensing (RS) images. Usually impulse bursts do not corrupt RS images due to hardware malfunction when the images are formed onboard the RS system carrier. Instead, the corruption happens during the data transmission phase when the impulse noise (that can be intensive enough in some cases) occurs [1]. In most cases a radio-frequency line is used for the information transferring. If the communication line is poor (i.e., the interference signals have power that is about the power of the broadcasting signal), impulse bursts can be observed on the transmitted images even through a digital line that uses noise-stable coding. However, the probability of the occurrence of a burst is much higher if an analog transmission line is used because such lines usually do not have any special means for the protection against the interference of the parasite signal.

An example of such situation is the old satellite image transmission standard, the so-called "automatic picture transmission" (APT) mode. The reason for its wide use nowadays is that freely distributed images from various satellites such as NOAA, GEOSat, Meteosat, "SICH," "Kosmos," "Okean" etc. are transmitted using this standard. The resolution of such freely distributed images is lower than the one of commercially distributed or military pur- pose RS images. The low complexity and cost of the reception devices and the great value of the RSinformation contained in such APT-mode transmitted RS images make, however, them very attractive for consumers. Thus, hundreds if not thousands people worldwide receive images from satellites in APT-mode and face the distortion of these images by impulse bursts [2].

The main reason for the occurrence of bursts is the interference of frequency modulated carrying signal with the signals from other sources of emission. The APT-mode frequency band lies in a widely used range of frequencies. The central frequency of the APT-mode is about $137 \mathrm{MHz}$. Besides, the influence of the interference becomes more essential when the useful signal power is decreased. Such situation is observed when the reception point drifts with respect to the satellite to the reception zone border (during the communication session). This results in an increased probability of impulse distortion in the top and bottom rows of the image. The rowwise transmission of images also determines the typical horizontal orientation of the compact impulse bursts (CIB) (see Fig.1a).

Reliable elimination of CIBs by means of standard robust filters can be achieved only by using sliding windows that are large enough. However, e.g., the $5 \times 5$ median filter leads to significant smearing of useful information, as can be seen in Fig.1c. The center weighted median filter (CWMF) [3] pre- 
serves details better than the median but, unfortunately, it can not remove impulse bursts as efficiently. This can clearly be seen in Fig.1b, which shows the image that was obtained using the center weight 3.

Some known techniques adapted for the removal of impulse bursts are based on the following basic approach: to replace only those pixels that are classified as bursts. That is, the filtering procedure can be split into two practically independent operations: the detection of the pixels distorted by bursts and the calculation of the restored values for the pixels classified as bursts. However, the known techniques for removing intensive impulsive noise are designed for noise models that are too simple for the considered case $[4,5]$. For example, for the restoration of the distorted pixels, standard robust order statistic filters (mostly the median filter) are used. This leads to errors in the cases when more than half of the pixels inside the sliding window are corrupted by bursts.

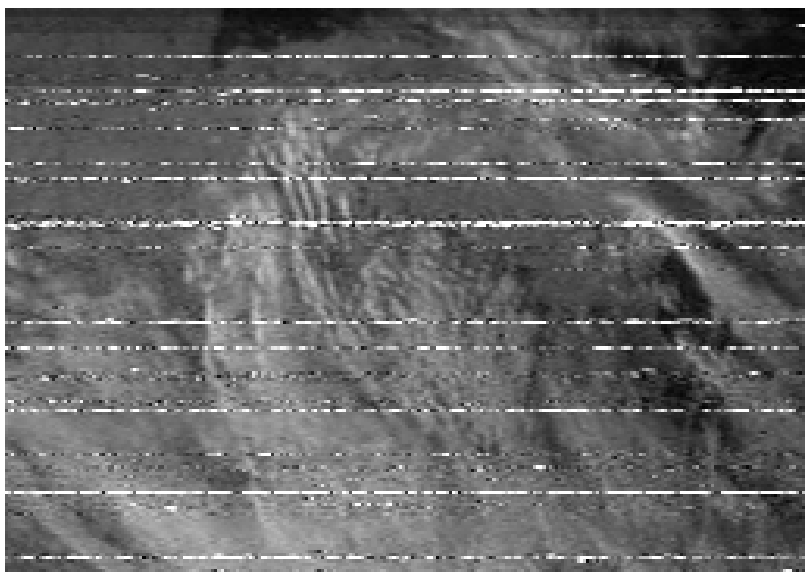

(a)

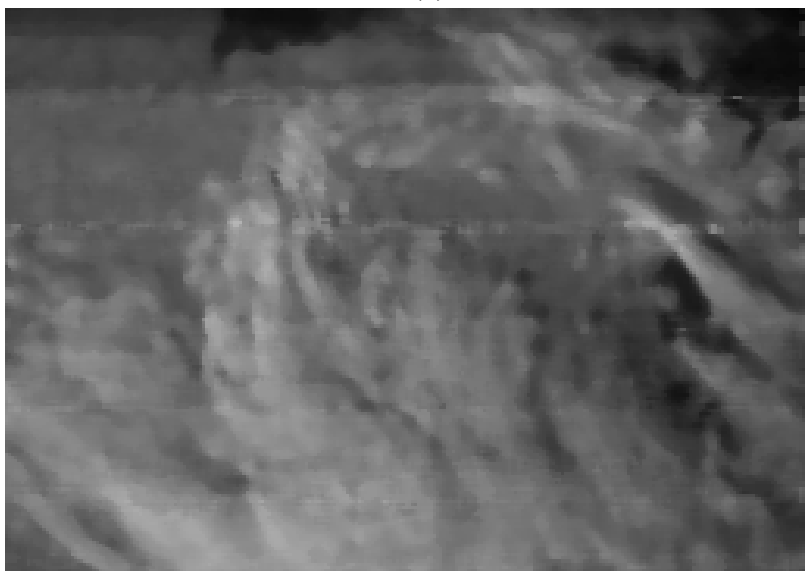

(c)
The noise model closest to CIBs can be found from the paper of Abreu and Mitra [6] concerning streak removal. The authors modify the method earlier proposed in [4] and suppose that if more than $30 \%$ of the pixels in a row are classified as impulses, then the entire row is considered to be a streak and the row is subject to replacement. However, although a CIB can corrupt the entire row this is not typical. More often a CIB corrupts tens of consecutive pixels in a row (see Fig. 1a). Thus, applying the method [6] for the CIB removal results in a considerable distortion for a large number of pixels in satellite image rows that are in fact not corrupted by bursts. Another difficulty for the method in [6] is the restoration of distorted pixels when several consecutive rows are corrupted by CIBs (see Fig.1d). In addition, besides CIBs, the satellite images also contain multiplicative and/or additive noise, which causes problems for the techniques [4-6].

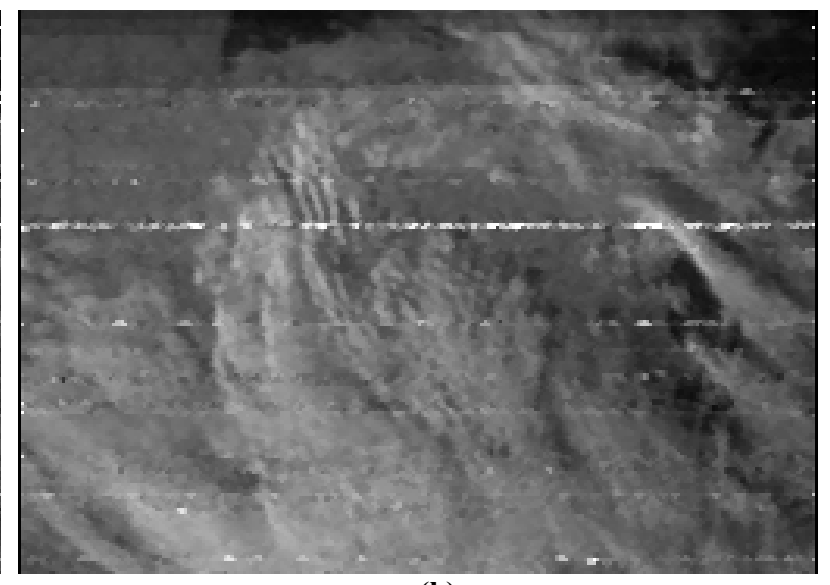

(b)

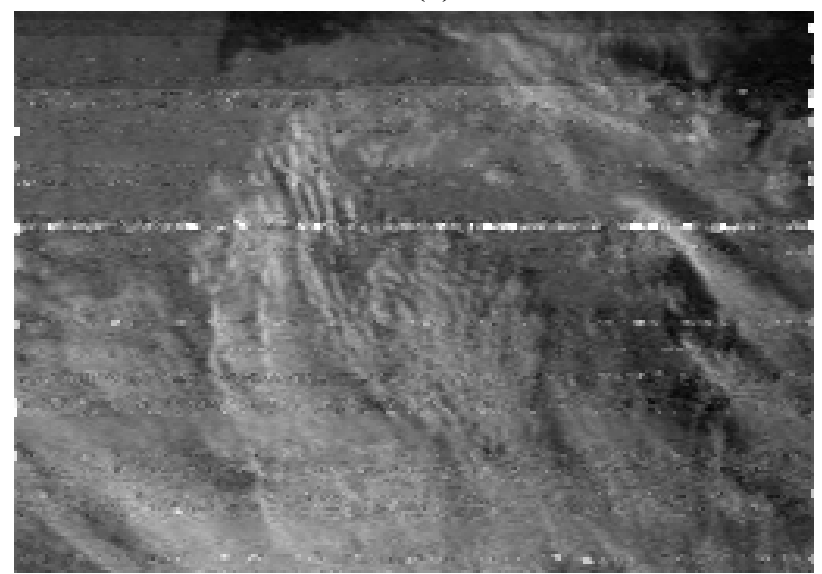

(d)

Fig.1 - Visual results of CIB removal in optical satellite image (a) by: (b) the CWMF 3x3 with the central element weight 3, (c) standard median filter 5x5, (d) the special method proposed by Abreu, Mitra et al.

In our paper [2], the possibility of using trainingbased soft morphological filters for the CIB removal was studied. Although practically full elimination of CIBs with simultaneous preservation of uncorrupted information was achieved, the method is computationally quite heavy to use. Especially, the search for suitable soft morphological filters may take some time. Hence, it is still desirable to study the compromise between the degree of information distortion (especially for texture and small size objects with horizontal orientation) and the degree of CIB removal. 
A multistage method for the CIB detection and removal in the presence of both additive and multiplicative noise was proposed in our paper [7]. This approach eliminates CIBs rather reliably and at the same time appropriately preserves information contained in the images. Its drawback is slight misclassification of small size objects as CIBs. To get rid of this drawback the modified version of the method [7] is proposed below. It consists of several stages and uses spatial features permitting to distinguish CIBs and horizontally oriented small size objects.

\section{COMPACT IMPULSE BURST MODEL}

To get an idea what the impulse bursts are, let us first analyze satellite images that were received by the Kalmykov Center of Radiophysical Sensing of Earth (Kharkov, Ukraine) via the APT telecommunication channel from Ukrainian satellite "Sich-1." A part of an optical image is presented in Fig.1a and a fragment of a radar image in Fig.2. As can be seen, several fragments in many rows are corrupted by impulse bursts, and the lengths of such fragments are rather different.

Moreover, sometimes such fragments occur in two consecutive rows. It can also be observed that in some pixels of the considered fragments, the values are maximal (i.e., 255 in the 8 -bit image representation used) while some pixel values differ from 255 but still remain "impulsive" with respect to the values that can be predicted for the satellite images from their local analysis.

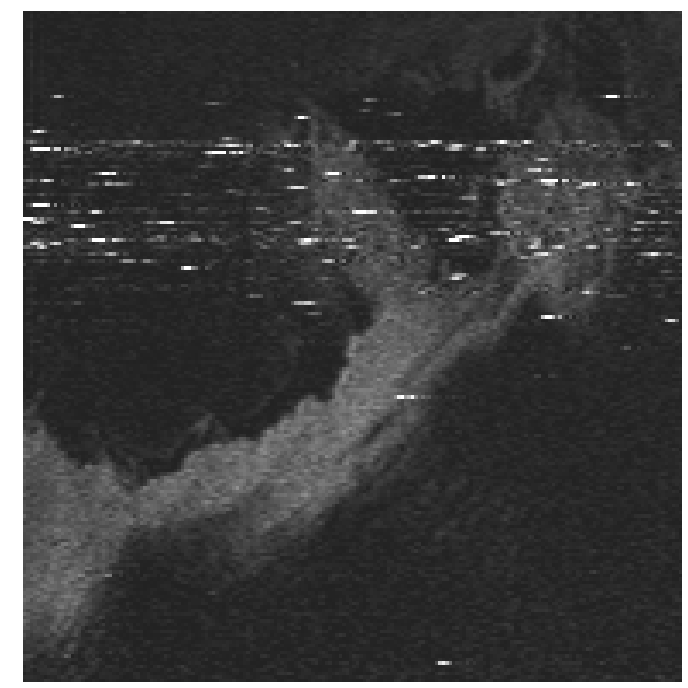

Fig.2 -Real radar satellite image with CIBs

A detailed study of the burst properties for many real images has brought us to the following main conclusions [2]. First, the percentage of pixels corrupted by impulse bursts can reach up to $11 \%$. By estimating the statistical characteristics of the bursts, we found that the mean of the burst fragments varied from 160 to 190 with cut-off effects observed for bright burst pixels. The bursts contained not only stochastic noise component but also some kind of quasi-sinusoidal component. These properties can clearly be seen in Fig.3 where one rowwise section of the optical satellite image in Fig. 1a is given.

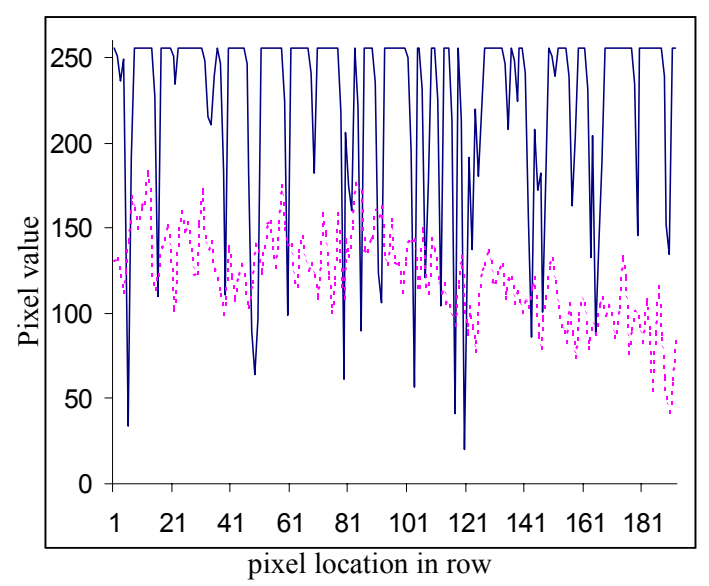

Fig.3 - Neighboring rows of the optical image shown in Fig.1,a: row 169 (dashed) that is highly corrupted by bursts; row 170 (solid) that is not corrupted.

The fluctuative noise that corrupts the pixels not corrupted by CIBs leads to additional difficulty for the burst elimination. Instead of weak additive noise typical for optical images (Fig.1a), multiplicative noise is present in the real radar image (Fig.2). The probability density function for this noise is Gaussian with unit mean and relative variance that can in our cases be as high as 0.05 .

All aforementioned properties of CIBs and fluctuative noise have been taken into account when generating the noise model for the test images used for training soft morphological filters with the aim of CIB removal in [2]. The image/noise model given here is a slightly modified version of the one given in our earlier paper [2]. Below we use it for comparison purposes to evaluate the performance of the considered methods.

The model is based on Markov stochastic process with two states. The states are used to determine which samples belong to impulse bursts. The transition probability from "no-burst-state" to "burststate" is $P_{0 \rightarrow 1}^{i m p}$, and the transition probability from "burst-state" to "no-burst-state" is $P_{1 \rightarrow 0}^{\text {out }}$.

If a sample does not belong to an impulse burst (i.e., the process is in "no-burst-state"), then the sample is corrupted by the aforementioned fluctuative noise as

$$
g(i, j)=u \cdot f(i, j)+n,
$$

where $g(i, j)$ and $f(i, j)$ are the pixels of the corrupted and the original image, respectively, $u$ is the multiplicative noise component with unit mean and 
relative variance $\sigma_{\mu}^{2}$, and $n$ is the additive noise component with zero mean and variance $\sigma_{n}^{2}$.

On the other hand, if a sample belongs to a CIB (i.e., the process is in "burst-state"), then the corrupted sample value is obtained using the following formula (the image is given as a 1-D array since in real situation the image is corrupted by bursts when it is transferred row by row)

$$
\begin{aligned}
g^{L}(j)= & \operatorname{round}\left\{f^{L}(j)+\zeta_{j} \beta_{b} \cdot\left(\sin \left[\left(j-k_{s}^{b}\right) \varpi_{b}+\varphi_{b}\right]+\right.\right. \\
& \left.\left.+\gamma_{b}\right)+\xi_{j}\right\}, \quad j=\overline{k_{s}^{b}, k_{e}^{b}}
\end{aligned}
$$

where $g^{L}(j), f^{L}(j)$ are the values of the corrupted and original images (in the one-dimensional array), respectively, $k_{s}^{b}, k_{e}^{b}$ are the one-dimensional array indices of the first and the last pixel of the CIB, respectively, $\beta_{b}, \varpi_{b}$ are the amplitude and the frequency of the harmonic component of the CIB, $\varphi_{b}$ denotes the phase of the harmonic component, $\zeta_{j}, \xi_{j}$ are the Gaussian multiplicative and additive components of the CIB (with unit and zero means and variances ${\sigma_{\mu i}}^{2}, \sigma_{n i}{ }^{2}$ ), respectively, and $\gamma_{b}$ is the pedestal (constant value) added to the harmonic component. Rounding is to the nearest non-negative integer less than or equal to 255 .

As the aim is to provide a smooth change in the harmonic component values at the beginning and at the end of each separate CIB, the starting phase value is $\varphi_{b}=-\pi / 2$. The values $\beta_{b}, \varpi_{b}$ are constant during one separate CIB, and they are generated as uniformly distributed random values from the intervals $\left[\beta_{\min } ; \beta_{\max }\right]$ and $\left[\varpi_{\min } ; \varpi_{\max }\right]$, respectively, for different CIBs. The pedestal value $\gamma_{b}$ is also constant for all pixels within one CIB. Its value is $\gamma_{b}=p_{c}+\left(\beta_{b}-\beta_{\text {min }}\right) /\left(\beta_{\max }-\beta_{\min }\right)$ where $p_{c}$ is from 0.4 to 1 . Although $\beta_{b}, \varpi_{b}$, and $\gamma_{b}$ are constant for all values in one CIB, new values for $\beta_{b}, \varpi_{b}$, and $\gamma_{b}$ are generated if the burst is long enough, that is, if $j>k_{s}^{b}+2 \pi / \varpi_{b}$. The values $\zeta_{j}, \xi_{j}$ vary from pixel to pixel.

Fig.4 shows a noise free test image containing different classes of information: large size homogeneous objects and small size objects with different shapes and contrasts to background as well as textural regions. Fig.5 gives the image in Fig. 4 corrupted by the proposed noise model with CIBs and multiplicative fluctuative noise typical for satellite side-look aperture radar (SLAR) images. The parameter values used equal to those estimated from the real images. The multiplicative noise variance was $\sigma_{\mu}^{2}=0.02$ and the transition probabilities were
$P_{0 \rightarrow 1}^{i m p}=0.0007$ and $P_{1 \rightarrow 0}^{\text {out }}=0.011 \quad\left(\sigma_{n}^{2}=0\right)$. As the result, the probability that a pixel belongs to a burst was about 0.05 . The parameters for the CIBs were $\sigma_{\mu i}{ }^{2}=0.37, \quad \sigma_{n i}{ }^{2}=400, \quad p_{c}=0.5, \quad \beta_{\min }=1, \quad \beta_{\max }=180$, $\varpi_{\text {min }}=0.02$, and $\varpi_{\text {max }}=0.85$. As can be seen, the artificial noise in the corrupted test image is very close to the noise in the real images given in Fig.1a and Fig.2.

The artificial test image pair presented in Figures 4 and 5 gives us an opportunity to evaluate quantitatively the performance of different filtering methods. As the error criterion between the filtered and the noise-free test image, it is possible to use any criteria that can be calculated using the filtered and noisefree images as parameters. In this paper we have used the mean square error (MSE) and the peak signal-to-noise ratio

$$
\mathrm{PSNR}=10 \log _{10}\left(255^{2} / \mathrm{MSE}\right) .
$$

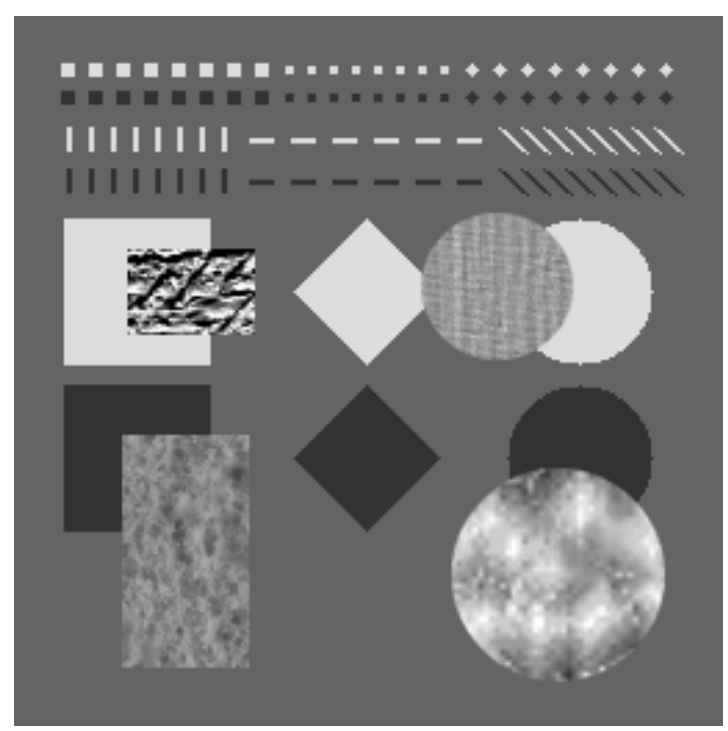

Fig.4 - The noise free test image

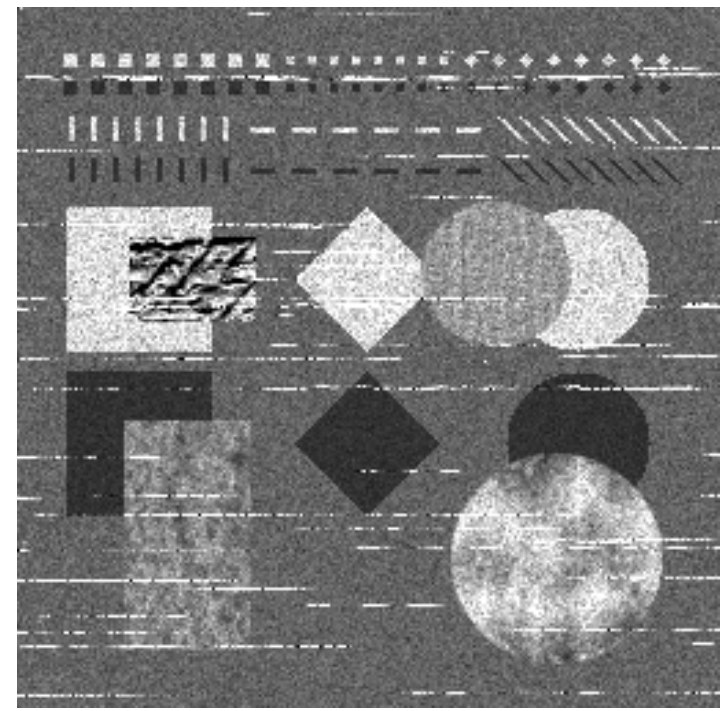

Fig.5 - The test image presented in Fig.4 corrupted accordingly to the proposed CIB noise model 
The fact that our test image contains all information classes that are typical for real remote sensing images ensures objectivity when estimating the filtering quality. In addition, CIB-free real satellite remote sensing images were corrupted by artificial CIBs according to the proposed CIB model. The obtained test images also let us to estimate the probability of correct and false CIB detection for the considered algorithm

\section{PROPOSED APPROACH}

At the first stage of the proposed method, the ordered samples (for the given sliding window) are grouped so that the neighboring order statistics belong to the same group according to the $2 \sigma$-criterion.

More formally, the condition for any two neighboring order statistics belong to one group in the presence of a mixture of multiplicative and additive noise is the following. The $(k+1)$ 'th order statistic $x_{(k+1)}$ (i.e., the element with the rank $k+1$ ) belongs to the group that includes the $k^{\prime}$ th order statistic $x_{(k)}$ (i.e., the element with the rank $k$ ) if the condition $x_{(k+1)}-x_{(k)} \leq x_{(k)} \cdot 2 \sigma_{\mu}+2 \sigma_{n}$ is satisfied, where $\sigma_{\mu}$ and $\sigma_{n}$ are the standard deviations of the multiplicative and additive noise, respectively.

This condition is written in a general form and it assumes that for given image one knows a priori or pre-estimates the statistical characteristics of additive and/or multiplicative noise, i.e. the relative variance $\sigma_{\mu}{ }^{2}$ and the variance $\sigma_{n}{ }^{2}$.

Among the formed groups, two groups are especially important for the further analysis: the group which contains the central pixel (CP) of the window, and the dominant group (DG), that is, the group with the greatest number of elements among all formed groups.

The necessity to minimize the false detection probability for the specified situations brought us to introduce additional parameters to the formula for the decision-making [7]. The new parameters consider the estimation of the structural features of objects. The modified formula for the determination of a CIB in the central pixel is

$$
\begin{gathered}
\Omega_{i j}^{B S}=M_{i j}^{m a p} \vee M_{i j}^{M V} \vee \neg M_{i j}^{h m g} \wedge \\
\wedge \neg M_{i j}^{H T} \wedge M_{i j}^{D} \wedge M_{i j}^{s t r}
\end{gathered}
$$

In the formula, $M_{i j}^{M V}$ indicates whether the $\mathrm{CP}$ value exceeds the greatest possible value for a given image. The parameters $M_{i j}^{H T}$ and $M_{i j}^{D}$ indicate, respectively, if the CP belongs to a semitone on the object border and if the size of the DG is sufficiently large when compared to the size of the group to which the CP belongs in order that the $\mathrm{CP}$ can be classified to belong to a CIB. The parameter $M_{i j}^{h m g}$ is the indicator for the absence of heterogeneity inside the sliding window. It has the value "true" if all samples inside the window belong to one group after the $2 \sigma$-segmentation. The analytical expressions and more detailed description for the parameters $M_{i j}^{M V}$, $M_{i j}^{H T}, M_{i j}^{h m g}$, and $M_{i j}^{D}$ are given in [7].

The introduced new parameters are the following. The parameter $M_{i j}^{\text {map }}$ informs that in the "burst map" (if it is already generated) the $\mathrm{CP}$ already was classified as a CIB at earlier pass(es), but it could not be correctly replaced. Such map is initially obtained at the first pass and then, at consequent passes, it is corrected. Each next pass (iteration) gives additional information concerning whether or not the sliding window central pixel is corrupted by CIB and what other neighbor pixels correspond to CIB.

The parameter $M_{i j}^{s t r}$ indicates whether the structural features of the object to which the $\mathrm{CP}$ belongs are similar to the structural features of a CIB. The value of $M_{i j}^{s t r}$ is determined by the expression

$M_{i j}^{s t r}=\neg G_{i j}^{v r t} \wedge\left(G_{i j}^{H B} \vee G_{i j}^{S} \vee G_{i j}^{B N}\right) \wedge \neg G_{i j}^{B O}$,

where $G_{i j}^{v r t}$ is the indicator of the CP object covering the number of rows that exceeds the maximum possible number for present CIB, $G_{i j}^{H B}$ is the indicator of a one-row horizontal CIB, $G_{i j}^{S}$ is the indicator of mainly one-row horizontal CIB, $G_{i j}^{B N}$ indicates if the $\mathrm{CP}$ has neighboring pixels that are corrupted by bursts, and $G_{i j}^{B O}$ indicates whether the CP belongs to a bright (dark) point object or to some small size horizontal object.

The formation of these low level structural indicators is carried out (if necessary for the calculation of $M_{i j}^{\text {str }}$ ) using enough simple operations which, however, take into account a priori probability of burst in the CP that is determined with the help of "burst map".

Quantitative results obtained for the test image in Fig. 5 have shown that more than $80 \%$ corrupted by CIB have been correctly detected (the Abreu and Mitra's method produces only about $60 \%$ of correct detection but about 4 times larger amount of false detection). The advantage of the proposed method is that for pixels corrupted by CIBs that have not been correctly detected the noisy values usually only slightly differ from the corresponding pixel true values. 
If the CP is corrupted by a CIB (i.e., when $\Omega^{B S}$ is true), the value of the $\mathrm{CP}$ is replaced by the weighted average of the DG elements. The weights are formed so that the elements located spatially inside the $3 \times 3$ neighborhood of the CP (and not classified as CIBs) have weight 2 and all other elements have weight 1 .

Table 1 shows some quantitative results of using well known robust rank order statistic (ROS) filters for processing the artificial test image (Fig.5). The performance of some of the studied filters is also illustrated visually in Fig.1.

The best compromise between the detail preservation and the removal of CIBs and multiplicative noise was obtained by the CWM-filter with the sliding window size $3 \times 5$ (columns $x$ rows) and the central element weight 5 . The sliding window $3 \times 5$ was preferable for the CIB removal also when soft morphological filters were used [2].

Table 2, on the other hand, gives the quantitative results of processing the same artificial test image (Fig.5) by some specialized methods [2,6]. Most of these methods assume a two-phase procedure for noise removal: the main stage (i.e., CIB removal only) and the post-processing stage intended for the suppression of residual fluctuative noise. The only exception is the soft morphological filter that was trained to remove both the CIBs and the fluctuative noise simultaneously. The other soft morphological filter used here was trained to remove only the CIBs. The rather intensive residual multiplicative noise was suppressed at the post-processing stage [2].

Table 1. Quantitative results of filtering the test image by well known robust rank order statistic (ROS) filters. The test image was corrupted by artificial CIBs and multiplicative noise with $\sigma_{\mu}^{2}=0.02$ (Fig.5).

\begin{tabular}{|c|c|c|c|c|c|c|c|}
\hline & \multirow{2}{*}{ Filtering type } & \multicolumn{2}{|c|}{$3 \times 3$} & \multicolumn{2}{|c|}{$3 \times 5$} & \multicolumn{2}{|c|}{$5 \times 5$} \\
\hline & & MSE & PSNR & MSE & PSNR & MSE & PSNR \\
\hline & No filtering & 745.5 & 19.4 & 745.5 & 19.4 & 745.5 & 19.4 \\
\hline & Median filter & 242.9 & 24.3 & 283.9 & 23.6 & 331.1 & 22.9 \\
\hline పี & CWMF-3 & 216.9 & 24.7 & 237.5 & 24.4 & 283.0 & 23.6 \\
\hline 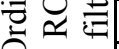 & CWMF-5 & 431.0 & 21.8 & 202.4 & 25.1 & 255.0 & 24.1 \\
\hline & Wilcoxon filter & 357.8 & 22.6 & 336.5 & 22.9 & 324.1 & 22.8 \\
\hline
\end{tabular}

Table 2. Quantitative results of the CIB removal by known methods and by the proposed algorithm with postprocessing stage. The test image was corrupted by artificial CIBs and multiplicative noise with $\sigma_{\mu}^{2}=0.02$ (Fig.5).

\begin{tabular}{|c|c|c|c|c|c|c|}
\hline \multirow{3}{*}{ CIB removing process } & \multicolumn{6}{|c|}{ Post-processing } \\
\hline & \multicolumn{2}{|c|}{ No processing } & \multicolumn{2}{|c|}{ Lee filter (5x5) } & \multicolumn{2}{|c|}{ DCT-filter $(8 \times 8)$} \\
\hline & MSE & PSNR & MSE & PSNR & MSE & PSNR \\
\hline $\begin{array}{l}\text { Abreu et al. [6] streak removing method with the } \\
\text { thresholds } T_{1}=15 ; T_{2}=30 ; T_{3}=50 ; T_{4}=80\end{array}$ & 392.0 & 22.2 & 228.7 & 24.5 & 202.2 & 25.1 \\
\hline 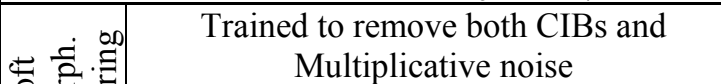 & 190.0 & 25.4 & \multicolumn{4}{|c|}{ Not used } \\
\hline Trained to remove CIBs only & 272.8 & 23.8 & 164.1 & 26.0 & 140.3 & 26.7 \\
\hline CIB removal by the proposed method & 325.1 & 23.0 & 135.7 & 26.8 & 114.3 & 27.6 \\
\hline
\end{tabular}

One of the most suitable specialized methods for the CIB removal proposed by Abreu et al. [6] also does not suppress fluctuative noise and needs to be followed by post-processing at the multiplicative noise suppressing stage.

For multiplicative noise suppression we chose two detail preserving filters: the Lee filter [8] suitable for speckle removal and a modification of the DCT-filter designed to remove multiplicative noise [9]. As can be seen from Table 2, the $8 \times 8$ DCT-filter suppresses multiplicative noise better than the Lee filter (with the most preferable $5 \times 5$ window size) in all cases.

Quantitative results for the artificial test image show that the Abreu method [6] (even supplied by the DCT-filter post-processing stage) does not pro- duce better results than the CWMF-5 with the $3 \times 5$ window. Visual results for the real satellite image shows that after the Abreu filter some CIBs are remaining (see Fig.1d).

The training-based soft morphological filtering [2] for the CIB removal produces the best results among the known methods (Table 2). The soft morphological filter trained to remove CIBs only, supplied by a post-processing stage had more than $1 \mathrm{~dB}$ better PSNR results when compared to the soft morphological filter trained to remove both the CIBs and the multiplicative noise simultaneously. However, visually there was not much difference in the results. The method introduced in this article for the CIB detection/removal together with the DCT-filter postprocessing stage provides additional $1 \mathrm{~dB}$ increase in 
the PSNR.

The quantitative results obtained for the artificial test image are in coherence with the visual inspection of the CIB removal for the real optical and radar

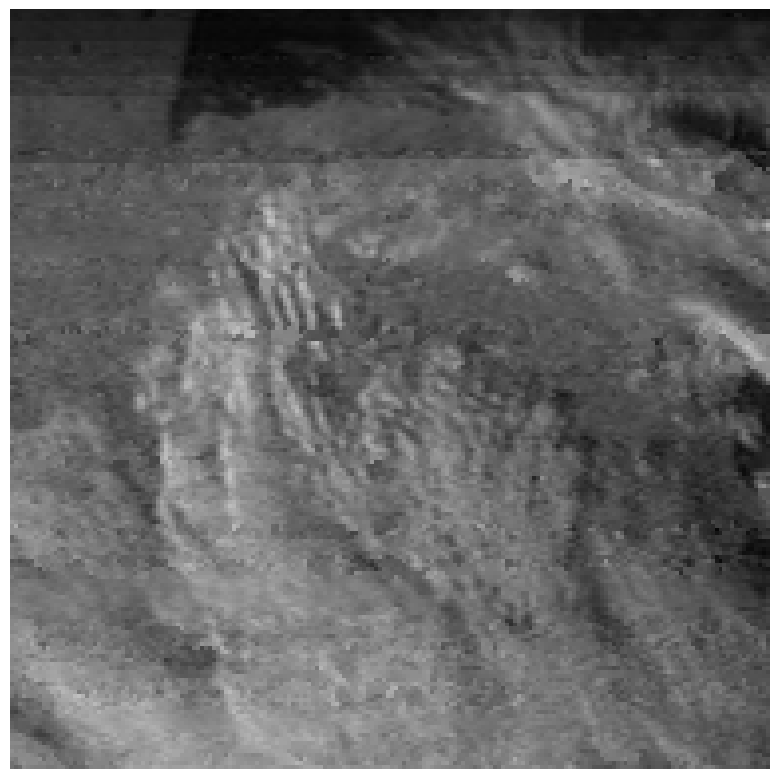

a) satellite images in Fig.1a and Fig.2. Fig.6 shows the result of the CIB removal by the proposed method. As can be seen, practically all CIBs are removed while important information remains undistorted.

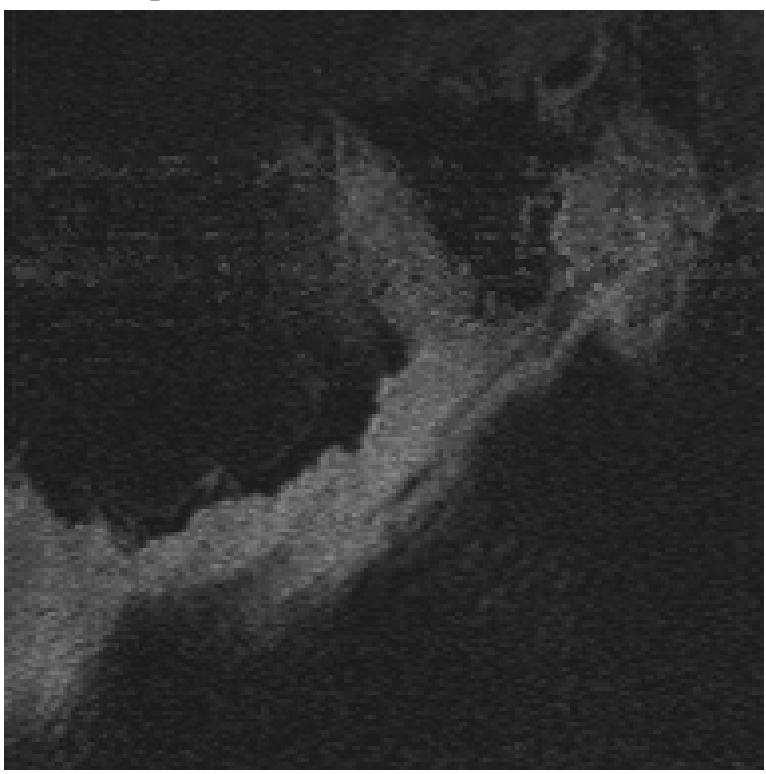

b)

Fig.6 - CIB removal by means of the proposed method from optical (a) and radar (b) satellite images presented in Fig.1, a and Fig.2, respectively.

\section{CONCLUSION}

Reasons for the CIB presence and the characteristics of the CIBs in real APT-mode transmitted satellite images were studied. An advanced model of satellite images corrupted by CIBs and fluctuative noise was developed, and the corresponding test image pairs needed in the quantitative evaluation of the filters were created.

A new approach for the CIB removal based on the multistage procedure of the CIB detection and on detail preserving CIB removal was given. The detection method utilizes estimates of the structural characteristics of the CIBs. It was shown through experiments that the proposed method produces better PSNR than the other existing methods. The advantages of the proposed method are also demonstrated visually for the real satellite images.

\section{REFERENCES}

[1] J.A. Richards. Remote Sensing Digital Image Analysis. An Introduction. Springer-Verlag, Berlin, Germany. 1986. 281 p.

[2] P. Koivisto, J.T. Astola, V.P. Melnik, V.V. Lukin, O.V. Tsymbal. Removing impulse bursts from images by training-based filtering, EURASIP Journal on Applied Signal Processing 3 (2003). pp. 223-237.

[3] I. Pitas, A.N. Venetsanopoulos. Nonlinear Digital Filters: Principles and Applications, Kluwer Academic Publishers, Boston, 1990.
[4] E. Abreu, M. Lightstone, S.K. Mitra, K. Arakawa. A new approach for the removal of impulse noise from highly corrupted images, IEEE Trans. on Image Processing 6 (5) (1996). pp. 1012-1025.

[5] How-Lung Eng, Kai-Kuang Ma, Noise adaptive soft-switching median filter, IEEE Trans. Image Processing 10 (2) (2001). pp. 242-251.

[6] E. Abreu, S.K. Mitra. A simple algorithm for restoration of images corrupted by streaks, IEEE Trans. on Image Processing (1996). pp. 730-733.

[7] O.V. Tsymbal. Removal of impulse noise on radar images that appear during satellite information receiving, Air-space Technique and Technology, 14 (1999). pp.132-135. (in Russian).

[8] J.S. Lee. Digital image enhancement and noise filtering by use of local statistics, IEEE Trans. on Pattern Analysis and Machine Vision 2 (1980). pp. 165-168.

[9] K.O. Egiazarian, V.P. Melnik, V.V. Lukin, J.T. Astola. Local transform-based denoising for radar image processing. Nonlinear Image Processing and Pattern Analysis XII, SPIE Proceedings vol. 4304, San Jose, CA, USA, 2001, pp. 170-178. 


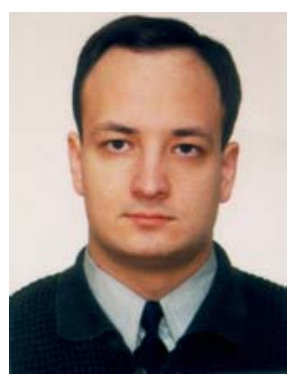

Oleg $V$. Tsymbal born in 1974, graduated from National Aerospace University (KhAl) of Kharkov, Ukraine, in 1998 and received Diploma of Computer Science. In 2003 he received the Candidate of Technical Science degree from National Aerospace University, Kharkov, in multichannel radar image processing. Since 1992, 1998 he is a associate Research Fellow and Research Fellow, respectively, in Kalmykov Center for Radiophysical Sensing of Earth National Academy of Sciences a National Space Agency of Ukraine. His research interests include digital image processing/filtering and remote sensing data interpretation.

\section{Volodymyr V. Lukin was} born in 1960 in Belarus. He graduated in 1983 from the faculty of Radioelectronic Systems of Kharkov Aviation Institute (now National Aerospace University), Kharkov, Ukraine, and received Diploma with honor in radioengineering.

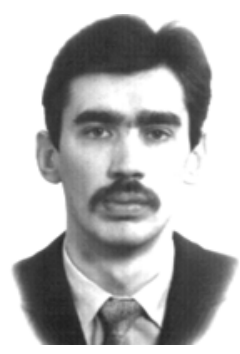

Since then he has been with Dept of Transmitters, Receivers and Signal Processing of the same faculty. He received Candidate of Technical Science Degree in Radioengineering in 1988, Senior Researcher Diploma in 1991, Doctor of Technical Science Degree in Remote Aerospace Research in 2002. Now he is the Associate Professor and ViceChairman of Dept of Transmitters, Receivers and Signal Processing in research work. His research interests include digital image processing/filtering and remote sensing data interpretation.

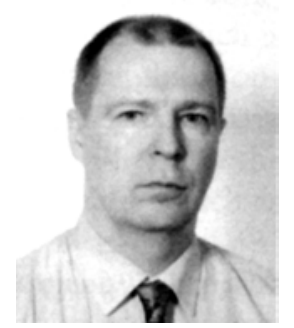

Pertti Koivisto received the M.S. and Licentiate degrees in mathematics from the University of Tampere, Finland, in 1984 and 1999, respectively. He received the Ph.D. degree in signal processing from Tampere University of Technology in 2000. Since 1981, he has held various teaching and research positions in mathematics and signal processing at the University of Tampere and at the Tampere University of Technology. Currently, he is a Senior Researcher at the Institute of Signal Processing, Tampere University of Technology. His main research interests include nonlinear signal and image processing and combinatorial optimization.
Volodymyr Melnik was born in 1966 in Khmelnitsky, Ukraine. He graduated from Kharkov Aviation Institute, Kharkov, Ukraine, in 1992 and received the M.S. degree in electrical engineering. Between 1992 and 1998, he was a Researcher and Senior Researcher at the same

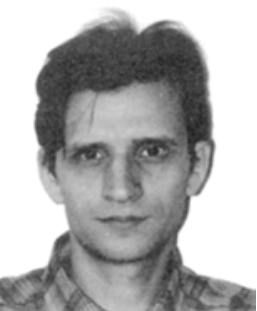
institute at the Department of Transmitting and Receiving Devices. In 1997, he received the Candidate of Technical Science degree from Kharkov Aviation Institute. From 1998 to 2000, he was with the Signal Processing Laboratory at the Tampere University of Technology, Tampere, Finland, where he received the Degree of Doctor of Technology in 2000. From 2001, he is with Nokia, Espoo, Finland. His research interests include image and signal processing and their applications. 